\title{
Lock-on-Chat: Boosting Anchored Conversation and Its Operation at a Technical Conference
}

\author{
Takeshi Nishida $^{1}$ and Takeo Igarashi ${ }^{1,2}$ \\ ${ }^{1}$ Department of Computer Science, The University of Tokyo \\ ${ }^{2}$ PREST JST \\ tnishida@ui.is.s.u-tokyo.ac.jp, takeo@acm.org
}

\begin{abstract}
This paper introduces a text-based chat system designed to support conversations anchored to specific locations of shared images and reports our experience in operating it at a technical conference. Our system is unique in that it focuses on supporting communications scattered around among multiple images, while other systems for anchored conversations are designed for deeper discussions within a single document. Our system was used in a technical conference as a space for anchored conversations over presentation slides and we observed that audiences actively participated in discussions during the presentation. The detailed chat $\log$ was also useful for both audiences and presenters.
\end{abstract}

\section{Introduction}

Most synchronous and asynchronous online communications are still primarily based on texts as seen in instant messaging, BBS, and online chat. This is because textbased communication has several advantages over other communication methods such as videos or voices. One reason is that texts can show long conversation history in a single view, which is difficult with streaming media. Another reason is that texts make it possible to have simultaneous multiple conversation sessions and also to interleave them with other activities such as web browsing. Finally, they require less equipments and mental preparation.

Some text based communication systems support communications anchored into documents. Shared annotation systems (e.g. Microsoft Office Web Discussions) provide a web-based front-end for digital annotations created by readers and writers. These systems not only offer opportunities for asynchronous conversations around documents, but also encourage collaborative writing. Anchored conversations [3] is a synchronous version of such communication, providing a text-chat window anchored to a specific point in a document.

These systems are appropriate for deep discussions within a document. However, they are not suitable for communications over multiple documents because it is difficult to monitor the whole communication when messages are scattered around various locations. In a study of a shared web annotation system, Cadiz et al. reported the users' slow response time using the system [2]. Improving the notification system is one way to facilitate the conversation [1], yet it is not appropriate for synchronous systems. 
We have built a system called Lock-on-Chat, to support fluid transition among conversational threads anchored to multiple images. The key idea is to gather all activities and provide an interactive summary of these activities. This paper describes its user interface and reports our experience with the system at a 3-day technical conference.

\section{Design Details}

Lock-on-Chat allows image sharing and anchored conversation over shared images. Figure 1 shows its screenshot. The user can upload still images to the server by dragand-drop to the client window and the uploaded images are shared by all clients. Once images are shared, the user can easily switch to different images by clicking the corresponding thumbnail in the list. Initiating a new anchored conversation is also easy; all the user has to do is to click the point of interest in an image and type the first message. We call such an anchored thread, lock-on.

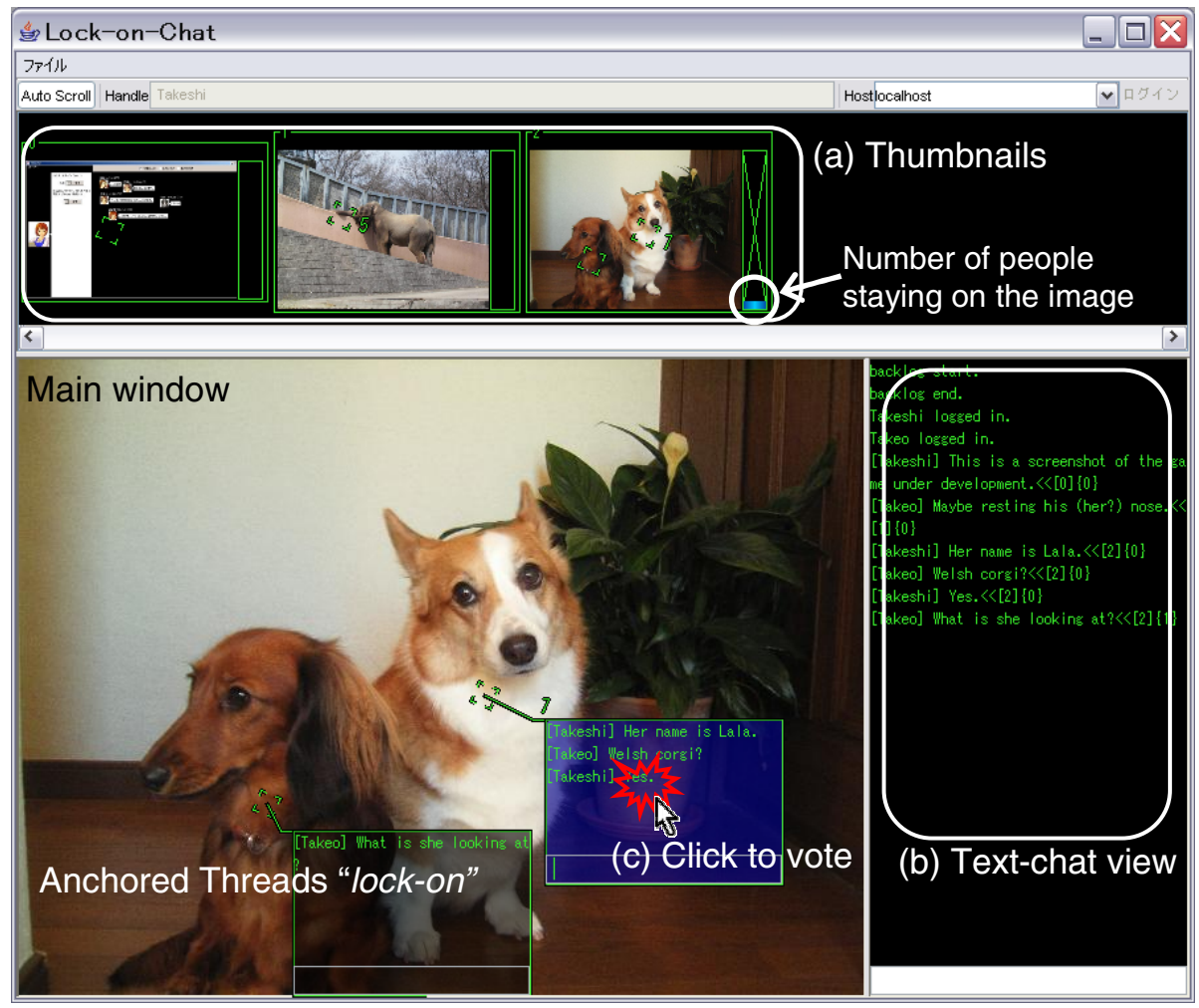

Fig. 1. Screenshot of Lock-on-Chat 
Lock-on-Chat provides three features to boost anchored conversations. First, a thumbnail indicates the location of anchored threads and the number of people looking at the image (Figure 1(a)). It helps the user to detect where hot conversations are taking place at a glance.

Second, all messages are gathered and shown in chronological order just like an ordinary text-chat client in the right pane (Figure 1(b)). The user can jump to a lockon by simply clicking the corresponding message. Messages not locked on to an image are also shown in this part; conversations are not required to be anchored.

Third, each lock-on has a simple vote function (Figure 1(c)). To vote for a lock-on, the user clicks repeatedly on it. The vote count is shown beside the lock-on and on the thumbnail. This can be used for many purposes; for example, choosing from several candidates, attracting people to the lock-on, or just expressing the excitement.

\section{Experience at a Technical Conference}

We operated our prototype system at a technical conference called WISS 2004. WISS (Workshop on Interactive Systems and Software) is an annual workshop in Japan, focusing on user interface technologies [6]. It is a single-track conference and has about 150 participants. Presentation sessions have been augmented by a chat system since WISS'97 and various chat systems have been tested since then. In the first trial by Rekimoto et al [5], Comic Chat [4] was operated, resulting in the strong support from participants for further trials.

During the presentations, most attendances were in the main conference room equipped with their own note PC. The contents of the main screen (presentation slides and demonstrations) were manually captured and uploaded to the server by an operator.

\subsection{Results and Observations}

98 people (about two-thirds of the entire participants) have connected to the chat server and sent messages at least once. Messages were sent 6.24 times/minute and images were uploaded 1.33 times/minute on average during the sessions. $48.4 \%$ of the messages were anchored to images, although the conversation was not restricted to be anchored. This heavy traffic indicates that people were able to follow and participate in the fast conversation scattered to a number of presentation slides.

For the audience, sharing the presentation slides was truly helpful. Many commented that easy access to previous slides was very helpful to follow the talk. In addition, simply having a slide at hand was helpful for people sitting a seat toward the back because the main screen was rather small compared to the room size. Moreover, some felt that they were concentrated on the presentation than usual. One reason could be that people were more attentive in order not to miss a chance to lock-on.

For the presenters, vote function seemed to be appealing. One presenter prepared several paths in the presentation and selected the branch to take during the talk according to the vote result. This attempt was successful, demonstrating the potential of dynamic, interactive presentation. The current system does not provide any assistance for the presenters, so the operator had to report the vote result each time. It is our 
future work to provide such functionality. Supporting the presenters to monitor the conversation would also enable them to answer questions during the presentation.

Chat log was also very beneficial to both the audience and the presenters. All captured slides (except the ones that were told as confidential by a presenter) were included in the published chat log. The chat log anchored to slides helped them to report the activities at the conference to their group and to review their presentations for improvement.

\section{Conclusion and Future Work}

We introduced the Lock-on-Chat system, which boosts anchored conversations with three additional features; rich thumbnails, text-chat view, and vote function. We have also reported its application to a technical conference as a chat system running in parallel with the presentation session. The results suggest that the proposed features are effective for communications over multiple documents. In addition, we have observed the importance of sharing the presentation slides and the need of supporting presenters in monitoring the conversation.

We are planning to enhance the visual effects to boost the conversation furthermore. For example, some visual effect for the vote count would attract people to the thread. We are also planning to provide assistance for presenters to monitor the conversation without controlling the GUI.

\section{References}

1. Brush, A. J. B. Bargeron, D. Jonathan, G. and Gupta, A. Notification for Shared Annotation of Digital Documents, In Proc of CHI2002.

2. Cadiz, J. Gupita, A. and Grudin, J. Using Web Annotations for Asynchronous Collaboration Around Documents, In Proc. of CSCW2000.

3. Churchill, E. F., Trevor, J., Bly, S., Nelson, L., and Cubranic, D. Anchored Conversations: Chatting in the Context of a Document, In Proc. of CHI2000.

4. Kurlander, D. Skelly, T. and Salesin, D. Comic Chat, In Proc. of SIGGRAPH96.

5. Rekimoto, J. Ayatsuka, Y. Uoi, and H. Arai, T. Adding Another Communication Channel to Reality: An Experience with a Chat-Augmented Conference, In the Conference Summary of CHI98.

6. WISS website available at http://www.wiss.org/ 